\title{
Peroneus Herniation with Superficial Peroneal Nerve Compression in an Adolescent Female: Case Report with Overview and Management
}

\author{
Daniel Sylvestre, MS*, Brittney Ehrlich, Max Cornell and Mark Seeley, MD \\ Orthopaedics Department, Geisinger Health, Danville, PA, USA
}

\begin{abstract}
A 13-year-old female presents with years of left lateral leg pain and a palpable protrusion, numbness on the dorsum of the foot, and paresthesia elicited by dorsiflexion of the left ankle. A diagnosis of superficial peroneal nerve (SPN) irritation/compression secondary to peroneal muscle herniation was made. When conservative measures failed, superficial peroneal nerve neurolysis with peritoneal fascial repair lead to a resolution of all symptoms and rapid return to activity.
\end{abstract}

\section{Keywords}

Lower extremity, Peroneus muscle, Herniation, Superficial peroneal nerve, Nerve compression, Adolescent, Herniation repair, Pediatric, Female

\section{Introduction}

Although extremity muscle hernias are common, symptomatic cases involving the peroneal muscles and concurrent irritation of the superficial peroneal nerve are rare. This report presents a successful surgical intervention as well as a review of relevant literature to aid in diagnosis and management despite a current lack of strong consensus on optimal treatment.

\section{Case Presentation}

A 13-year-old female presented with a several-year history of left lateral leg pain with numbness on the dorsum of the foot. Dorsiflexion of the ankle elicited a palpable protrusion on the lateral aspect of the leg which was associated with pain and occasional numbness or paresthesia. MRI and ultrasound were consistent with peroneal muscle herniation, with concern for superficial peroneal nerve (SPN) irritation and/or compression. A trial of nonoperative management consisting of avoiding exacerbating activities and nonsteroidal anti-inflammatories did not reduce symptoms, and the family elected to proceed with surgical intervention - superficial peroneal nerve neurolysis with fascial repair.

\section{Surgical technique and outcome}

After the patient was prepped, a $4 \mathrm{~cm}$ incision was made at the location where the hernia was palpable, directly over the lateral aspect of the left peroneal tendons. Blunt dissection revealed the peroneal tendons herniating

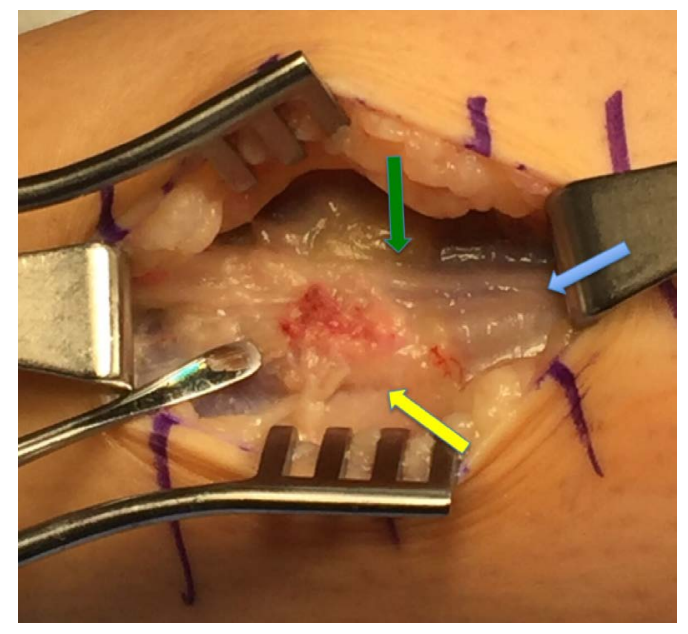

Figure 1: Intraoperative photograph of peroneus longus muscle belly (blue arrow), and fascial defect with herniation (yellow arrow) compressing the superficial peroneal nerve (green arrow).

*Corresponding author: Daniel Sylvestre, MS, Orthopaedics Department, Geisinger Health, Danville, PA, USA

Accepted: November 18, 2019

Published online: November 20, 2019

Citation: Sylvestre D, Ehrlich B, Cornell M, Seeley M (2019) Peroneus Herniation with Superficial Peroneal Nerve Compression in an Adolescent Female: Case Report with Overview and Management. J Orthop Surg Tech 2(2):80-83 
Citation: Sylvestre D, Ehrlich B, Cornell M, Seeley M (2019) Peroneus Herniation with Superficial Peroneal Nerve Compression in an Adolescent Female: Case Report with Overview and Management. J Orthop Surg Tech 2(2):80-83

through a fascial defect about $2.5 \mathrm{~cm}$ in length. Directly superior to the herniation, the SPN was located and observed to be compressed (Figure 1). The fascial defect was lengthened $1 \mathrm{~cm}$ on either side and the SPN was fully explored to ensure no compression or neuroma, and fibrotic tissue was removed (Figure 2). The two branches of the superficial peroneal nerve were explored to confirm decompression (Figure 3). The fascia was then closed, taking great care to prevent entrapment, and leaving an approximately $5 \mathrm{~mm}$ opening where the nerve exits the fascia (Figure 4). The nerve was in good health with no ischemia and good capillary refill in this region. After fascial closure and copious irrigation, 2-0 Vicryl suture was used subcutaneously followed by 4-0 Monocryl, Steri-Strips, and a soft dressing. $0.25 \%$ Marcaine was injected around the nerve, and the patient was then successfully extubated.

Upon discharge, the patient was weight-bearing with the

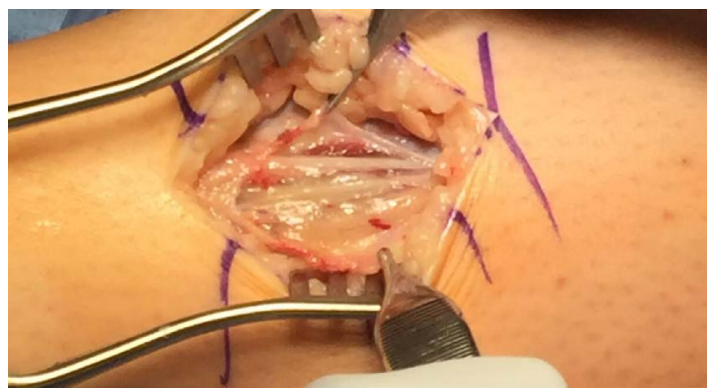

Figure 2: Intraoperative photograph of opened fascial defect with fibrotic tissue removed.

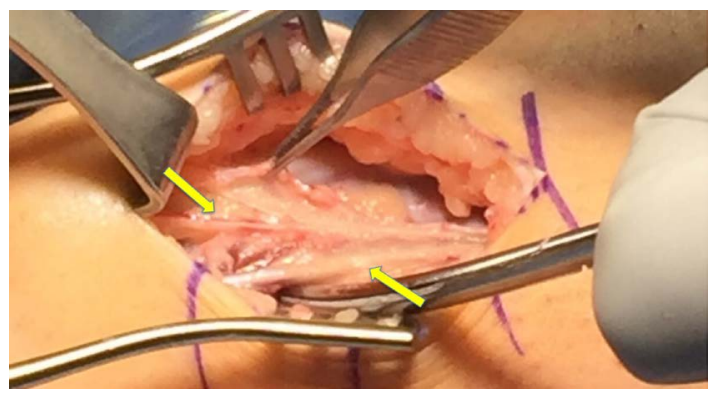

Figure 3: Intraoperative photograph of the two branches of the superficial peroneal nerve observed to be free of compression (yellow arrows).

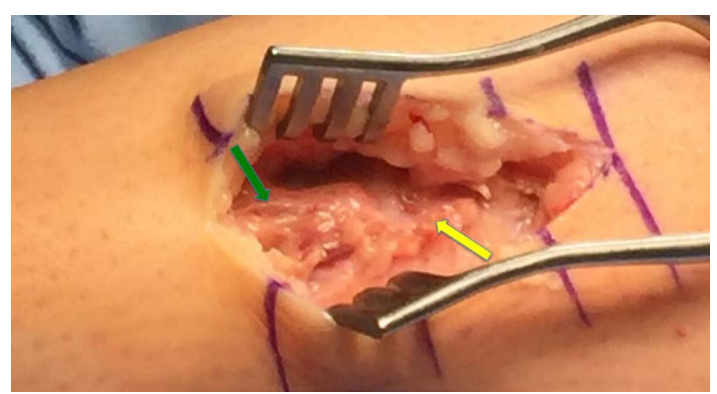

Figure 4: Intraoperative photograph showing fascial defect after closure (yellow arrow), and two branches of the superficial peroneal nerve exiting the $0.5 \mathrm{~cm}$ fascial opening (green arrow). use of a boot. She was seen at follow-up two weeks later at which time she had no pain or paresthesia and her only complaint was swelling. She was instructed to continue ice and elevation and begin walking without the boot at home. By 8 months postoperatively, she was completely asymptomatic, had full strength and range of motion, and had returned to nearly all activities. The patient remains asymptomatic and without recurrence of hernia upon present reporting 18 months post-operatively.

\section{Overview of extremity muscle hernias: Epidemi- ology and diagnosis}

Although the legs are the most common location of extremity muscle herniation, symptomatic cases are minimally represented in the literature $[1,2]$. Such hernias are caused by focal fascial defect, either congenital or acquired. Congenital cases may be due to simple fascial weakness or occurring at sites of normal perforation such as those for nerves or vessels [3]. Acquired cases are typically secondary to trauma. For both types, higher intracompartmental pressure from muscle hypertrophy increases risk, particularly in athletes [4]. The most commonly involved muscle of the leg is the tibialis anterior, with hernia of the peroneus muscles rarely reported [3-5]. However, when they do occur, peroneus muscle hernias are commonly associated with compression of the superficial peroneal nerve (SPN), as hernias of the lateral compartment tend to occur at the site where the SPN normally perforates the deep fascia [6].

Clinical presentation of lower extremity muscle hernia may include a visibly palpable bulge, soft tissue mass or subcutaneous nodule, which may be singular or multiple, and may or may not be easily reducible. Patient complaints may include discomfort, weakness, pain, or neuropathy which may worsen with standing or exertion. Hernias may be elicited with movements which require contraction of involved muscles. Patients with asymptomatic hernias may present with only cosmetic concerns or concern of a tumor, and incidental discovery during unrelated examination have also been reported [7]. Differential diagnosis includes hematoma, varicosity, angioma, arteriovenous aneurysm, epidermoid cyst, lipoma, schwannoma, tumor, sprain or fracture, pseudohernia, and central neuropathy $[4,7-9]$. Ultrasound or MRI can help confirm the diagnosis, and both can be improved using dynamic imaging techniques which allow for movements required to elicit the hernia [10-12]. Ultrasonography may be the best initial imaging choice due to relative ease and cost, although MRI is superior in visualizing musculofascial demarcation and quantification of the defect.

\section{Anatomy of SPN and diagnosis of compression}

The SPN provides motor innervation to the peroneus longus and brevis muscles, as well as cutaneous innervation to the anteroinferior leg and part of the dorsum of the foot and toes. It is a terminal branch emerging at the bifurcation of the common fibular nerve in the popliteal fossa. It then travels inferiorly along the fibula through the lateral compartment (or the anterior compartment in 
Citation: Sylvestre D, Ehrlich B, Cornell M, Seeley M (2019) Peroneus Herniation with Superficial Peroneal Nerve Compression in an Adolescent Female: Case Report with Overview and Management. J Orthop Surg Tech 2(2):80-83

roughly $25 \%$ of the population) [13]. Although the precise location of emergence is quite variable, it typically pierces the crural fascia into the superficial fascia and subcutaneous fat on the anterolateral aspect of the leg, approximately $10-12 \mathrm{~cm}$ proximal to the tip of the lateral malleolus [14]. The SPN divides into the medial and intermediate dorsal cutaneous nerves (MDCN, and IDCN). This typically occurs after the nerve emerges into the superficial fascia but may occur before emergence in approximately $28 \%$ of the population. More distally, both the MDCN and IDCN cross anterior to the talocrural joint and divide into dorsal digital nerves, supplying portions of digits 1 to 3 , and 3 to 5 , respectively. In rare instances, either the MDCN or IDCN may be absent, in which case branches of either the saphenous or sural nerves will cover the cutaneous innervations [15]. It is important to appreciate the variable course of the SPN and its branches to effectively interpret clinical signs of compression, and to avoid iatrogenic injury during surgical intervention. Diagnostic nerve blocks using small amounts of lidocaine into the superficial and deep fascial compartments can be useful for identifying any anatomic variability, whether other nerve branches are involved, and the exact location of compression [16].

\section{Treatment options and outcomes}

Muscle hernia of the leg is often successfully treated with conservative therapy including rest, activity restrictions, and compression stockings. However, surgical referral is warranted for patients with moderate to severe symptoms, and those including nerve involvement. There is no consensus regarding optimal surgical treatment. Described treatments include decompressive fasciotomy, direct primary fascial repair (as was performed in this case), fascial patch grafting or stripping using autologous fascia lata, and the use of synthetic mesh. Attempts to address excessive volume of the underlying muscle have included partial muscle excision and injections of sclerosing agents, local anesthetic, or botulinum toxin $[17,18]$. Each approach involves exploration and dissection to release the entrapped SPN, but each operation has disadvantages. Grafting may require additional or longer incisions and risks foreign body incorporation or undesirable adherence. Anatomical fascial repair, as employed in this case, requires close post-operative observation due to risks of acute or chronic compartment syndrome or hernia recurrence. Longitudinal fasciotomy minimizes the risk of compartment syndrome, but may result in incomplete resolution of pain, cosmetic concerns due to increased muscle bulging, neuromuscular damage including weakness or dysesthesias, or venous disease from disruption of the calf muscle pump [16]. Regardless of procedure, outcomes of similar cases of extremity muscle hernias where nerves are subjected to traction or compressive forces indicate that elective surgery be performed sooner rather than later to minimize the risk of persistent nerve pathology [12]. Treatment is time sensitive because pathological changes in the nerve may continue due to continued inflammation or vascular degeneration, even after harmful forces have been removed $[19,20]$. Thus, surgically releasing the nerve may not immediately relieve symptoms, although it is important for preventing further damage. Still, the incidence of resolution of pain after decompression is approximately $85 \%$, if diagnosed correctly. Perioperative drugs such as gabapentin, desipramine, topiramate, vitamin $\mathrm{C}$, and topical analgesics can be useful for managing pain and aiding recovery [16].

\section{Conclusion}

Due to the lack of strong consensus on optimal treatment for symptomatic peroneal muscle herniation with SPN compression, appreciating the anatomy and pros/cons of various treatment options is critical. In this case of an adolescent female, SPN neurolysis with fascial repair achieved excellent outcomes- full resolution of pain at 2 weeks follow-up, full resolution of symptoms and near full return to activities by the 8-month follow-up, as well as no hernia recurrence at 18 months post-op.

\section{References}

1. Bloem JJ (1976) The treatment of muscle hernias by fascial splitting. Br J Plast Surg 29: 291-294.

2. Kitchin ID, Richmond DA (1943) Multiple Muscle Herniae. Br Med J 1: 602-603.

3. Braunstein JT, Crues JV (1995) Magnetic resonance imaging of hereditary hernias of the peroneus longus muscle. Skeletal Radiol 24: 601-604.

4. Berglund HT, Stocks GW (1993) Muscle hernia in a recreational athlete. Orthop Rev 22: 1246-1248.

5. Mellado JM, Pérez del Palomar L (1999) Muscle hernias of the lower leg MRI findings. Skeletal Radiol 28: 465-469.

6. Garfin S, Mubarak SJ, Owen CA (1977) Exertional anterolateral-compartment syndrome. Case report with fascial defect, muscle herniation, and superficial peroneal-nerve entrapment. J Bone Joint Surg Am 59: 404-405.

7. Alfageme F, Morales V, García C, et al. (2011) Transfascial muscular hernia: An unusual cause for a 'hide and seek' subcutaneous nodule. Dermatol Online J 17: 4.

8. Ceyhan AM, Chen W, Yener M, et al. (2010) Bilateral tibialis anterior muscle herniation simulating a soft tissue tumour in a young amateur football player. Australas J Dermatol 51: 142-144.

9. Davies JA (1979) Peroneal compartment syndrome secondary to rupture of the peroneus longus. A case report. JBJS 61: 783.

10. Tyson S, Subhas N (2010) Radiologic case study. Gastrocnemius fascial defect and muscle herniation. Orthopedics 33: 785.

11. Zeiss J, Ebraheim NA, Woldenberg LS (1989) Magnetic resonance imaging in the diagnosis of anterior tibialis muscle herniation. Clin Orthop Relat Res 249-253.

12. Donovan A, Rosenberg ZS, Cavalcanti CF (2010) MR imaging of entrapment neuropathies of the lower extremity. RadioGraphics 30: 1001-1019.

13. Canella C, Demondion X, Guillin R, et al. (2009) Anatomic Study of the Superficial Peroneal Nerve Using Sonography. American Journal of Roentgenology 193: 174-179.

14. Adkison DP, Bosse MJ, Gaccione DR, et al. (1991) Anatomical variations in the course of the superficial peroneal nerve. J Bone Joint Surg Am 73: 112-114. 
Citation: Sylvestre D, Ehrlich B, Cornell M, Seeley M (2019) Peroneus Herniation with Superficial Peroneal Nerve Compression in an Adolescent Female: Case Report with Overview and Management. J Orthop Surg Tech 2(2):80-83

15. Agthong S, Huanmanop T, Sasivongsbhakdi T, et al. (2008) Anatomy of the superficial peroneal nerve related to the harvesting for nerve graft. Surg Radiol Anat 30: 145-148.

16. Bregman PJ, Schuenke MJ (2016) Commentary on the diagnosis and treatment of superficial peroneal (fibular) nerve injury and entrapment. Journal of Foot and Ankle Surgery 55: 668-674.

17. Nguyen JT, Nguyen JL, Wheatley MJ, et al. (2013) Muscle hernias of the leg: A case report and comprehensive review of the literature. Can J Plast Surg 21: 243-247.
18. Yoo MJ, Kim D, Lee J, et al. (2007) Poster 211: Injection of Botulinum Toxin as a Treatment for Superficial Peroneal Nerve Entrapment Caused by Muscle Hernia: A Case Report. Archives of Physical Medicine and Rehabilitation 88: E70.

19. Nobel W (1966) Peroneal palsy due to hematoma in the common peroneal nerve sheath after distal torsional fractures and inversion ankle sprains. J Bone Joint Surg Am 48: 1484-1495.

20. Golshani SD, Lee C, Sydorak R (1999) Symptomatic forearm muscle hernia: Repair by autologous fascia lata inlay. Ann Plast Surg 43: 204-206. 